\title{
Temperature distribution in magnetized neutron star crusts
}

\section{The effect of a strong toroidal component}

\author{
U. Geppert ${ }^{1}$, M. Küker ${ }^{2}$, and D. Page ${ }^{3}$ \\ 1 Max-Planck-Institut für extraterrestrische Physik, Giessenbachstrasse, PF1312, 85741 Garching, Germany \\ e-mail: urme@mpe.mpg.de \\ 2 Astrophysikalisches Institut Potsdam, An der Sternwarte 16, 14482 Potsdam, Germany \\ 3 Instituto de Astronomía, UNAM, 04510 Mexico D.F., Mexico
}

Received 16 December 2005 / Accepted 27 May 2005

\section{ABSTRACT}

\begin{abstract}
We continue the study of the effects of a strong magnetic field on the temperature distribution in the crust of a magnetized neutron star (NS) and its impact on the observable surface temperature. Extending the approach initiated in Geppert et al. (2004), we consider more complex and, hence, more realistic, magnetic field structures but still restrict ourselves to axisymmetric configurations. We put special emphasis on the heat blanketing effect of a toroidal field component. We show that asymmetric temperature distributions can occur and a crustal field consisting of dipolar poloidal and toroidal components will cause one polar spot to be larger than the opposing one. These two warm regions can be separated by an extended cold equatorial belt. As an example we present an internal magnetic field structure which can explain, assuming local blackbody emission, both the X-ray and optical spectra of the isolated NS RXJ 1856-3754, the hot polar regions dominating the X-ray flux and the equatorial belt contributing predominantly to the optical emission. We investigate the effects of the resulting surface temperature profiles on the observable lightcurve which an isolated thermally emitting NS would produce for different field geometries. The lightcurves will be both qualitatively (deviations from sinusoidal shape) and quantitatively (larger pulsed fraction for the same observational geometry) different from those of a NS with an isothermal crust. This opens the possibility to determine the internal magnetic field strengths and structures in NSs by modeling their X-ray lightcurves and spectra. The striking similarities of our model calculations with the observed spectra and pulse profiles of isolated thermally emitting NSs is an indication for the existence of strong magnetic field components maintained by crustal currents.
\end{abstract}

Key words. stars: neutron - magnetic fields - conduction - dense matter

\section{Introduction}

It has been understood since Greenstein \& Hartke (1983) that in presence of a sufficiently strong magnetic field, $\geq 10^{10} \mathrm{G}$, the surface temperature of a neutron star (NS) will not be uniform as is expected in the unmagnetized case. Anisotropy of heat transport, caused by both classical and quantum magnetic field effects, in the thin low density $\left(\rho \leq 10^{10} \mathrm{~g} \mathrm{~cm}^{-3}\right)$ upper layer, the so called envelope, results in a strongly reduced conductivity in the direction perpendicular to the field and an enhanced one along the field. As a result, the regions around the magnetic poles are expected to be significantly warmer than the regions around the magnetic equator. Page (1995) and Page \& Sarmiento (1996) applied the Greenstein \& Hartke formula to explain the lightcurves of the isolated thermally emitting NSs PSR 0833-45 (Vela), PSR 0656+14, PSR 0630+178 (Geminga) and PSR 1055-52, considering dipolar field configurations, and also the addition of a quadrupolar component, and including the General Relativistic curvature effects on photon trajectories. Much work has been dedicated to study the effects of the magnetic field on the properties of the NS envelope and crust (for reviews, see Yakovlev \& Kaminker 1994; Heyl \& Hernquist 1998; Ventura \& Potekhin 2001; Lai 2001). Due to the shallowness of the envelope, $\sim 100 \mathrm{~m}$, heat transport can be treated in the plane parallel approximation as a one dimensional problem in which the heat flux is purely radial with a, locally uniform, magnetic field having some arbitrary orientation with respect to the radial direction. The most recent studies (Potekhin \& Yakovlev 2001, henceforth "PY01"; and Potekhin et al. 2003) have included the best up to date transport coefficients and equation of state: they showed some deviations from the simple Greenstein \& Hartke formula and finally give us a reasonably accurate description of a magnetized NS envelope.

In presence of a sufficiently strong magnetic field, $\gtrsim 10^{12}-10^{13} \mathrm{G}$, the anisotropy of heat transport, simply due to the classical effect of Larmor rotation of the electrons, extends to much higher densities and can even be present within the whole crust. Recently, we have shown (Geppert et al. 2004, subsequently Paper I) that in cases where the field geometry in the crust is such that the meridional component of the field dominates over its radial component in a large part of the crust, as is the extreme case of a magnetic field entirely confined to the crust, the non uniformity of the temperature, previously considered to be restricted to the envelope, may actually extend to the whole crust. This modified crustal temperature results in a different surface temperature distribution than what the simple Greenstein \& Hartke model predicted. In contradistinction, in case the dipolar poloidal field has its currents localized only in the core of the star, i.e., the field in the crust is considered as the one of a vacuum dipole, the crust is practically isothermal and the non uniformity of the surface temperature is then only due to field effects in the envelope. This result, that the geometry of the magnetic field in the interior of the NS leaves an observable imprint at the surface, potentially allows us to study the internal 
structure of the magnetic field through modelling of the spectra and pulse profile of thermally emitting NSs.

There exist growing observational evidence that the anisotropy of the heat transport in the envelope alone, assuming an otherwise isothermal crust, can not explain the surface temperature distributions of some observed NSs. The seven X-ray dim isolated NSs (XDINSs), dubbed "The Magnificent Seven" (for reviews, see, e.g., Haberl 2004a,b; 2005) are nearby isolated NSs, all discovered in the X-ray band where they show a thermal spectrum. As a set they share several properties: 1) low X-ray absorptions imply small distances, $<200-500 \mathrm{pc} ; 2$ ) high blackbody temperatures deduced from the fit of their X-ray spectra indicate ages of the order of $10^{6} \mathrm{yr}$, possibly confirmed by measurements of proper motions which allow for tentative identification of their birth sites in clouds of the Gould's belt; 3) no detection of radio emission; 4) no detection of a hard Xray tail, typical of magnetospheric emission in active pulsars; 5) no association with a supernova remnant; 6) detected spin periods (in 5 cases) between 3 to $11 \mathrm{~s}$; 7) estimates of their surface dipolar field strength $B_{0}$ between $1-10 \times 10^{13} \mathrm{G}$, either from a measurement of the period derivative $\dot{P}$ (1 case) or the interpretation of broad absorption lines (in 5 cases) in the $\mathrm{X}$ ray spectrum as being proton cyclotron lines; 8) optical broad band photometric detections ( 5 cases) can be interpreted as being the Rayleigh-Jeans tail of a blackbody. However, these optical data are well above the Rayleigh-Jeans tail of the blackbody detected in the X-ray ("optical excess") and indicate the presence of an extended cold component of much larger area than the warm component observed in X-ray, the latter having an emitting radius $(\sim 3-5 \mathrm{~km})$ much smaller that the usually assumed radius of a NS ( $\sim 10-15 \mathrm{~km})$. Schwope et al. (2005) tried to fit the lightcurve of RBS 1223 and concluded that only a surface temperature profile with relatively small, about 4-5 km across, hot polar regions may explain the observations. Pons et al. (2002) and Trümper et al. (2004) arrived qualitatively at the same conclusion when they fitted the combined X-ray and optical spectrum of RX J1856.5-3754. In both cases, the smallness of the hot region is much below what can be reached by considering anisotropic heat transport limited to only a thin envelope. There are, however, not many possibilities to produce non uniform surface temperature distributions. Principially, rotation leads to an oblateness which enhances the thickness of the insolating outer layer at the equatorial belt making that region cooler than the poles (e.g. Geppert \& Wiebicke 1986). This effect is, however, even for the fastest rotating millisecond pulsars, too small and a fortiori neglibigle for those isolated thermally emitting NSs whose rotational period is in the order of seconds. Another way to heat the polar regions is the bombardment by ultrarelativistic charged particles as can be expected in radiopulsars (see Gil et al. 2003). Such heating is mostly confined to the polar caps whose angular radius can be roughly estimated as $\theta_{\mathrm{pc}} \sim(2 \pi R / c P)^{1 / 2}, R$ being the star's radius, $c$ the speed of light and $P$ the pulsar's rotational period, giving a polar cap radius $r_{\mathrm{pc}} \sim 0.46 P^{-1 / 2} \mathrm{~km}$ (for $P$ measured in $\mathrm{s}$ ), which is much smaller than the estimated size, a few kms, of the warm regions in the X-ray dim isolated NSs whose periods are in the range of 3 to $11 \mathrm{~s}$.

It should be mentioned, however, that there is a great uncertainity about the effects of a strong magnetic field on the thermal emission. A condensed surface, either liquid or solid, will cause an almost featureless spectrum, close to a blackbody, as observed for RX J1856.5-3754 (Brinkmann 1980; Turolla et al. 2004; Pérez-Azorín et al. 2005; van Adelsberg et al. 2005). Alternatively, a NS atmosphere containing only a small amount of hydrogen is also able to reproduce both the optical and X-ray spectrum of RX J0720.4-3125 without taking into account an additional thermal component (Motch et al. 2003).

The previous Paper I was devoted to demonstrate that a strong magnetic field can have significant effects on the crustal temperature distribution. We solved the stationary heat transport equation in Newtonian approximation and considered very simple, purely dipolar poloidal crustal field structures. Our goal here is to consider more realistic models in which the currents maintaining the poloidal magnetic field are distributed between the core and the crust and we also consider the possible presence of a strong toroidal component in the crust. We will still consider only axisymmetric, dipolar, magnetic field configuration. Moreover, we now perform a wholly general relativistic formulation of the heat transport and energy balance equations. Given the model uncertainities, as e.g. the crustal chemical composition and impurity content as well as the initial magnetic field configuration, the general relativistic effects are only a small correction, particularly for the stationary temperature distribution in the magnetized crust. For the construction of lightcurves and forthcoming cooling calculations, however, general relativistic effects are important and will therefore be taken into account from the beginning.

There exist also theoretical reasons that the presence of a strong toroidal field is very likely. In a series of papers Markay \& Tayler (1973, and references therein) showed that a stable magnetic field configuration needs a coexistence of poloidal and toroidal field components, having approximately the same strength. Recently, Braithwaite \& Spruit (2004) considered the stability of pure poloidal field geometries numerically in Ap stars and in magnetic white dwarfs and arrived at the same conclusion.

Little is known about the magnetic field structure in NSs which is very likely determined by processes during the protoNS phase and/or in a relatively short period after that epoch. A proto-NS dynamo (Thompson \& Duncan 1993) is unlikely to generate purely poloidal fields while differential rotation will easily wrap any poloidal field and generate strong toroidal components (Kluźniak \& Ruderman 1998; Wheeler et al. 2002). The magneto-rotational instability (Balbus \& Hawley 1991) also most certainly acts in proto-NSs (Akiyama et al. 2003) and results in toroidal fields from differential rotation (Balbus \& Hawley 1998). It is not yet explored whether the onset of supercondctivity in the core and the relatively fast crystallization of the largest part of the crust allows the field to relax into a stable (force free) state. However, it seems realistic to consider the effect of magnetic field configurations which consist of poloidal AND toroidal crustal components as well as of a star centered poloidal one. The relative strengths as well as the positions of the crustal components will depend on the - certainly varying creation processes of those fields and will therefore be varied in reasonable limits.

The paper is organized as follows: In the next section, Sect. 2, we define the axisymmetric field structures and consider the resulting $2 \mathrm{D}$ heat transport and energy balance equations. The next section, Sect. 3, presents our results for the crustal temperature distributions resulting from a large set of different field geometries and Sect. 4 studies the observable effects of the resulting surface temperature distributions, assuming simple isotropic blackbody emission. As an example we present a crustal magnetic field structure which allows us to reproduce the two temperature spectral fits of RX J1856.5-3754. A discussion of our results and conclusions are the objects of Sect. 5. 


\section{Heat transport and magnetic field}

We will assume that the heat transport through the core $\left(\rho_{\text {core }} \gtrsim\right.$ $1.6 \times 10^{14} \mathrm{~g} \mathrm{~cm}^{-3}$ ) is practically unaffected by the magnetic field. This assumption is justified by the very likely occurence of proton superconductivity which, in the usually assumed case that it is a type II superconductor, will confine the magnetic flux to fluxoids of microscopic size leaving the bulk of the core matter devoid of magnetic field ${ }^{1}$. As a consequence we consider the stellar core to be isothermal and we only need to define the magnetic field structure and calculate the temperature distribution in the crust. Moreover, we will still, in the present work, restrict ourselves to axisymmetric configurations.

\subsection{Structure of the magnetic field}

The assumption of axial symmetry suggests a decomposition of the NS magnetic field in a poloidal and a toroidal component and we will restrict ourselves to the lowest order terms, i.e., dipolar fields. As the magnetic field is divergence-free, its three component can be represented by two scalar potentials, $S$ and $T$ (Chandrasekhar 1961; Rädler 2000):

$\boldsymbol{B}=\boldsymbol{B}^{\text {pol }}+\boldsymbol{B}^{\text {tor }}=-\nabla \times(\boldsymbol{r} \times \nabla S)-\frac{\boldsymbol{r}}{R} \times \nabla T$.

The field represented by $S$ is called the poloidal field, the part expressed by $T$ the toroidal field. In case of axisymmetry the relation between the field components and the potentials, considering only the lowest order dipolar components, reduces to

$B_{r}=\frac{2 \cos \theta}{r^{2}} S(r, t)$,

$B_{\theta}=\frac{\sin \theta}{r} \frac{\partial S(r, t)}{\partial r}$,

$B_{\varphi}=\frac{\sin \theta}{r} T(r, t)$.

In this case $B_{r}$ and $B_{\theta}$ constitute the poloidal field while $B_{\phi}$ is the toroidal field.

The boundary conditions at the NS surface are such that the internal field matches smoothly an external potential field; an observer above the NS surface would see only a dipolar poloidal field, whatever internal field structure is present.

Basically we will consider three types of NS magnetic field:

1. The core field $\boldsymbol{B}^{\text {core }}$ is a dipolar field maintained by axisymmetric currents in the core. Then a vacuum-like dipolar field will penetrate the crust with components, in spherical coordiinates $(r, \theta, \varphi)$ oriented along the symmetry axis,

$$
\begin{aligned}
& B_{r}^{\text {core }}=B_{0}^{\text {core }} \frac{\cos \theta}{x^{3}}, \\
& B_{\theta}^{\text {core }}=-\frac{B_{0}^{\text {core }}}{2} \frac{\sin \theta}{x^{3}}, \\
& B_{\varphi}^{\text {core }}=0,
\end{aligned}
$$

where $B_{0}^{\text {core }}$ denotes the value of this component of the magnetic field at the magnetic pole on the surface of the star and $x=r / R_{\mathrm{NS}}$ the dimensionless radial coordinate, normalized on the NS radius $R_{\mathrm{NS}}$. This description of the core field in the crust assumes that it feels the crustal shell above the crustcore boundary as vacuum.

1 In case the protons form a type I superconductor, as has been proposed by Link (2003) and Buckley et al. (2004), this assumption would have to be revised.
2. The crustal poloidal magnetic field $\boldsymbol{B}^{\text {crust }}$ is maintained by toroidal currents circulating in the crust. As for the core field we will consider here only its largest scale component, which continues beyond the NS surface as a dipolar vacuum field. The field components read

$$
\begin{aligned}
& B_{r}^{\text {crust }}=B_{0}^{\text {crust }} \frac{\cos \theta}{x^{2}} s(x, t), \\
& B_{\theta}^{\text {crust }}=\frac{B_{0}^{\text {crust }}}{2} \frac{\sin \theta}{x} \frac{\partial s(x, t)}{\partial x},
\end{aligned}
$$

and

$$
B_{\varphi}^{\text {crust }}=0 \text {, }
$$

where $B_{0}^{\text {crust }}$ is - as for $B_{0}^{\text {core }}$ - the strength of this field component at the magnetic pole. We have introduced the normalized function $s=2 S / B_{0} / R_{\mathrm{NS}}^{2}$, which must satisfy the boundary conditions: $s=0$ at the crust-core boundary and $s=1$ with $\partial s / \partial x=-1$ at the surface.

3. The crustal toroidal magnetic field $\boldsymbol{B}^{\text {tor }}$ has only one non-zero component,

$B_{\varphi}^{\mathrm{tor}}=B_{0}^{\mathrm{tor}} \frac{\sin \theta}{x} t(x, t)$.

It is maintained by poloidal currents in the crust and vanishes at the NS surface. As we assume it does not penetrate the core, the potential $t$ must vanish at the surface and at the crustcore boundary while its maximum value within the crust is fixed to 1 .

The field is a superposition of the three field types described above. Notice that the toroidal field is symmetric with respect to the equatorial plane while the dipolar poloidal fields are antisymmetric and, hence, the total magnetic field is asymmetric. To maintain an antisymmetric field configuration, to a dipolar poloidal field one would have to add a quadrupolar toroidal $\boldsymbol{B}^{\text {tor }} \propto \sin \theta \cos \theta$, with all toroidal components with odd multipolarity vanishing.

Determination of the spin-down of a NS can give us an estimate of the strength of the dipolar poloidal field at the magnetic pole, $B_{0}$. In our models $B_{0}=B_{0}^{\text {crust }}+B_{0}^{\text {core }}$ : this gives us no information about either the possible values of $B^{\text {tor }}$, and the relative strengths of $B_{0}^{\text {core }}$ and $B_{0}^{\text {crust }}$, or about the shape of the two functions $s(x)$ and $t(x)$. There is thus a large degree of arbitrariness in the choice of the field structure, even within our restriction to the dipolar terms, and we will consequently consider many differente cases. We choose for $s(r)$ one of the models of Page et al. (2000) at an age $t \simeq 10^{5} \mathrm{yr}$ : in these time evolution models of crustal field, the field was not allowed to penetrate the stellar core due to proton superconductivity and the crustal currents sustaining the field were slowly migrating toward the high conductivity layers near the crust-core boundary. This gives us some confidence that our choice of $s(x)$ is not too unrealistic ${ }^{2}$. For the function $t(x)$ we use a Gaussian-like shape with a maximum at

$x_{\max }=x_{\text {core }}+\alpha \Delta x_{\text {crust }}$

where $x_{\text {core }}$ and $\Delta x_{\text {crust }}$ are the normalized core radius and crust thickness, resp., and consider three values of $\alpha: 0.2,0.5$, and 0.7 . The normalized Stokes function $s(x)$ and the three different $t(x)$ functions we will consider are plotted in Fig. 1. It is important to

2 Notice, however, that the models of Page et al. (2000) used a 1D numerical code and hence no $\theta$ dependance of the temperature in the crust, which is in contradiction with the results of Sect. 3. 


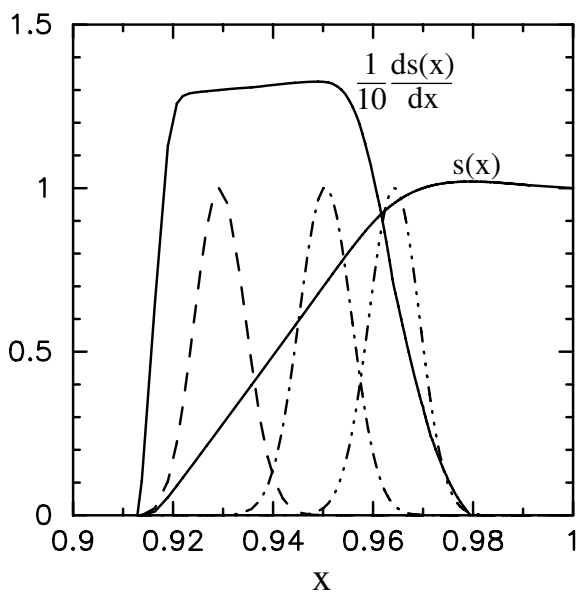

Fig. 1. Continuous lines: normalized Stokes function $s(x)$ and its derivative (scaled by a factor 10) used in this work, which generate $B_{r}$ (Eq. (2)) and $B_{\theta}$ (3) resp. Discontinuous lines: the three different normalized functions $t(x)$ we consider for the toroidal field, $B_{\varphi}$ (Eq. (4)).

notice that the maximum values of $B^{\text {core }}$ is $B_{0}^{\text {core }} / x_{\text {crust }}^{3} \simeq 1.3 B_{0}^{\text {core }}$, reached at the crust-core boundary, the maximum value of $B^{\text {tor }}$ is $B_{0}^{\text {tor }}$, in the crust at the point $x=x_{\max }$, while the maximum value of $B^{\text {crust }}$ is about 13 times $B_{0}^{\text {crust }}$, from its $\theta$ component (Eq. (3)), due to the fact that $s(x)$ must decrease from 1 at the surface to 0 at the crust-core boundary and hence has a large derivative $\partial s / \partial x \sim \Delta s / \Delta x_{\text {crust }} \approx 10$ since $\Delta x_{\text {crust }} \sim 0.1$, as shown in Fig. 1 . The large values of $B_{\theta}^{\text {crust }}$ compared to $B_{\theta}^{\text {core }}$ simply result from the fact that $B^{\text {crust }}$ being confined to the crust its magnetic flux is sqeezed within a thin shell while $B^{\text {core }}$ is allowed to expand within the core. The field lines of the poloidal components $B^{\text {crust }}$ and $B^{\text {core }}$ are, for each value of the longitudinal angle $\varphi$, contained in the $(r, \theta)$ plane and are illustrated in the left and central panels of Fig. 2 where the sqeezing of $B^{\text {crust }}$ inside the crust is clearly seen. For $B^{\text {tor }}$, its field lines are simply circles parallels to the equatorial plane and centered on the symmetry axis and the right panel of Fig. 2 shows the field intensity isolines in an $(r, \theta)$ plane.

\subsection{The two-dimensional heat transport}

The thermal evolution of the crust is determined by the energy balance equation which has, in axial symmetry and taking into account general relativistic effects, the following form:

$$
\begin{array}{r}
\frac{\mathrm{e}^{-\Lambda}}{r^{2}} \frac{\partial}{\partial r}\left(r^{2} F_{r} \mathrm{e}^{2 \Phi}\right)+\frac{\mathrm{e}^{2 \Phi}}{r \sin \theta} \frac{\partial}{\partial \theta}\left(\sin \theta F_{\theta}\right)= \\
\left(\mathrm{e}^{\Phi} C_{v} \frac{\mathrm{d} T}{\mathrm{~d} t}+\mathrm{e}^{2 \Phi} Q_{v}\right),
\end{array}
$$

where $T$ is the local temperature, $\mathrm{e}^{\Phi}, \mathrm{e}^{\Lambda}$ are the redshift and length correction factor, $F_{r}$ and $F_{\theta}$ are the local radial and meridional components of the heat flux and $r$ and $\theta$ the local coordinates. $Q_{v}$ and $C_{v}$ are the neutrino emissivity and specific heat, respectively, per unit volume. We will study stationary configurations and neglect neutrino energy losses, i.e., replace the righthand side of Eq. (13) by zero, and rewrite this equation as

$\frac{1}{x^{2}} \frac{\partial}{\partial \tilde{x}}\left(x^{2} \tilde{F}_{r}\right)+\frac{1}{x \sin \theta} \frac{\partial}{\partial \theta}\left(\sin \theta \tilde{F}_{\theta}\right)=0$

where $\partial / \partial \tilde{x} \equiv \mathrm{e}^{-\Lambda} \partial / \partial x$ and $\tilde{F}_{r, \theta} \equiv \mathrm{e}^{2 \Phi} F_{r, \theta} / R_{\mathrm{NS}}$.
In regions below the envelope, i.e. in the crust, the quantized motion of electrons transverse to the magnetic field lines doesn't play any role for the magnetic modification of the heat transport. The magnetic field acts via the Larmor rotation of the electrons, the relevant heat carriers, impeding on the heat transfer perpendicular to the crustal field. Introducing, as in Paper I, the components of the temperature gradient parallel and perpendicular to the magnetic field as well as the corresponding components of the heat conductivity tensor we arrive at the heat flux vector

$$
\begin{aligned}
& \mathrm{e}^{\Phi} \boldsymbol{F}=-\hat{\kappa} \cdot \boldsymbol{\nabla}\left(\mathrm{e}^{\Phi} T\right)=-\frac{\kappa_{0}}{1+\left(\omega_{B} \tau\right)^{2}} \\
& \quad \times\left[\boldsymbol{\nabla}\left(\mathrm{e}^{\Phi} T\right)+\left(\omega_{B} \tau\right)^{2} \boldsymbol{b}\left(\boldsymbol{\nabla}\left(\mathrm{e}^{\Phi} T\right) \cdot \boldsymbol{b}\right)+\omega_{B} \tau \boldsymbol{b} \times \boldsymbol{\nabla}\left(\mathrm{e}^{\Phi} T\right)\right],
\end{aligned}
$$

whose radial and meridional components are

$$
\begin{aligned}
\tilde{F}_{r}= & -\tilde{\chi}_{1} \frac{\partial \tilde{T}}{\partial \tilde{x}}+\tilde{\chi}_{2}\left(\frac{\partial \tilde{T}}{\partial \theta} \frac{s}{2 x^{4}} \frac{\partial s}{\partial \tilde{x}} \sin \theta \cos \theta-\frac{\partial \tilde{T}}{\partial \tilde{x}} \frac{s^{2}}{x^{4}} \cos ^{2} \theta\right) \\
& +\tilde{\chi}_{3} \frac{t}{x^{2}} \frac{\partial \tilde{T}}{\partial \theta} \sin \theta
\end{aligned}
$$

$$
\begin{aligned}
\tilde{F}_{\theta}= & -\tilde{\chi}_{1} \frac{1}{x} \frac{\partial \tilde{T}}{\partial \theta} \\
& +\tilde{\chi}_{2}\left(\frac{\partial \tilde{T}}{\partial \tilde{x}} \frac{s}{2 x^{3}} \frac{\partial s}{\partial \tilde{x}} \sin \theta \cos \theta-\frac{\partial \tilde{T}}{\partial \theta} \frac{1}{4 x^{3}}\left(\frac{\partial s}{\partial \tilde{x}}\right)^{2} \sin ^{2} \theta\right) \\
& -\tilde{\chi}_{3} \frac{t}{x} \frac{\partial \tilde{T}}{\partial \tilde{x}} \sin \theta
\end{aligned}
$$

where we have written $\tilde{T} \equiv \mathrm{e}^{\Phi} T$ and $\tilde{\chi}_{i} \equiv \mathrm{e}^{\Phi} \chi_{i}$.

The heat conductivity coefficients are:

$$
\chi_{1}=\frac{\kappa_{0}}{1+\left(\omega_{B} \tau\right)^{2}}, \chi_{2}=\frac{\kappa_{0}\left(\omega_{B_{0}^{p}} \tau\right)^{2}}{1+\left(\omega_{B} \tau\right)^{2}}, \chi_{3}=\frac{\kappa_{0}\left(\omega_{B_{0}^{t}} \tau\right)}{1+\left(\omega_{B} \tau\right)^{2}} .
$$

Note that the Larmor-frequencies $\omega_{B_{0}^{p, t}}$ in Eq. (18) are calculated with $B_{0}^{p}=B_{0}^{\text {core }}+B_{0}^{\text {crust }}$ in $\chi_{2}$ and with $B_{0}^{t}=B_{0}^{\text {tor }}$ for the "toroidal" heat conductivity coefficient $\chi_{3}$. The Larmor-frequency in the denominator of these coefficients is calculated by using the exact field strength at each point: $B^{2}=B^{\text {core }}(r, \theta)^{2}+B^{\text {tor }}(r, \theta)^{2}+$ $B^{\text {crust }}(r, \theta)^{2}$.

For a given magnetic field structure Eq. (13) is solved with the heat flux components of Eqs. (16) and (17) numerically using the same code which has been briefly described in Paper I until a stationary solution is found. The neutrino emissivity in the crust can be neglected for isolated NSs of that age. The inner boundary condition, at the crust-core boundary is $T\left(x=x_{\text {core }}, \theta\right)=T_{\text {core }}$, i.e. the core is assumed to be isothermal. For the outer boundary condition the argumentation given in Sect. 2.4. of Paper I remains valid and we proceed in the same way: we stop our integration at a density $\rho_{\mathrm{b}}=10^{10} \mathrm{~g} \mathrm{~cm}^{-3}$ and, at the corresponding $x_{\mathrm{b}}$ and for each $\theta$, the radial flux is given by $F_{r}\left(x_{\mathrm{b}}, \theta\right)=$ $\sigma_{\mathrm{SB}} T_{\mathrm{s}}^{4}$ where $T_{\mathrm{s}}$ is the surface (effective) temperature corresponding to the boundary temperature $T\left(x_{\mathrm{b}}, \theta\right)$ obtained with the " $T_{\mathrm{b}}-T_{\mathrm{s}}$ " resulting from the magnetized envelope models of PY01.

We use the same input physics as in Paper I for calculating the electron relaxation time $\tau$ and the same chemical composition of the crust. For the core of the star we here used the recent models of Akmal et al. (1998). We consider only a $1.4 M_{\odot} \mathrm{NS}$, with a radius $R_{\mathrm{NS}}=11.4 \mathrm{~km}, R^{\infty} \equiv R_{\mathrm{NS}} \cdot \mathrm{e}^{-\Phi}=14.2 \mathrm{~km}$, and $x_{\text {crust }}=0.914, \Delta x_{\text {crust }}=0.086$.

The essence of the effect of a strong magnetic field is that the heat flux $\boldsymbol{F}$ is forced to be almost aligned with the local field $\boldsymbol{B}$ 

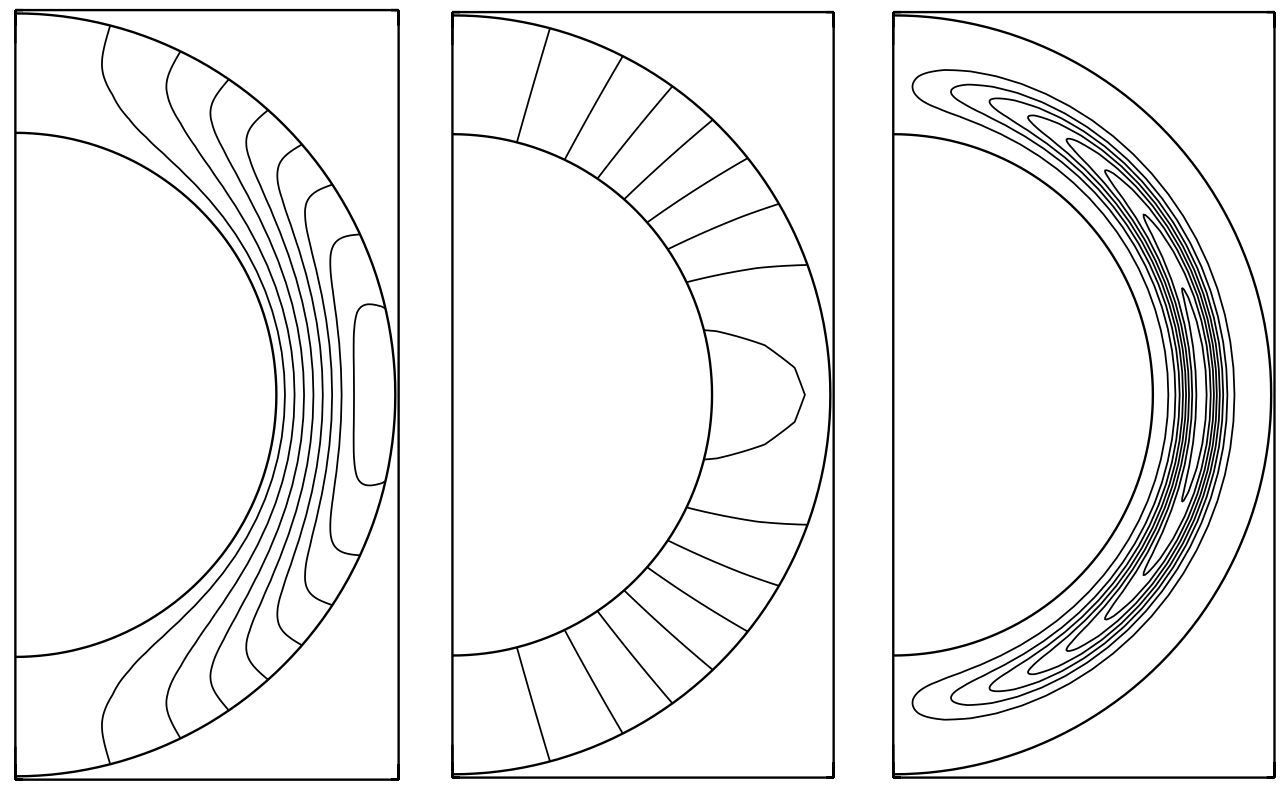

Fig. 2. Qualitative sketch, in the $(r, \theta)$ plane of the field geometries as considered here. Left and central panels show the field lines for the two types of poloidal fields: $B^{\text {crust }}$ in the left panel and $B^{\text {core }}$ in the central panel. The right panel show isolines of a toroidal field with its maximum in the middle of the crust $(\alpha=0.5)$.

when $\left(\omega_{B} \tau\right)^{2} \gg 1$ since then the component of the thermal conductivity tensor $\hat{\kappa}$ parallel to $\boldsymbol{B}$ is $\kappa_{\|}=\kappa_{0}$ while in the perpendicular directions it is $\kappa_{\perp}=\kappa_{0} /\left(1+\left(\omega_{B} \tau\right)^{2}\right) \ll \kappa_{\|}$. Values of $\omega_{B} \tau$ were plotted in Fig. 2 of Paper I. Notice that we do not calculate $F_{\varphi}$. By axial symmetry this component is independent of $\varphi$ but certainly not equal to zero, in spite of having $\partial T / \partial \varphi \equiv 0^{3}$. Since, for strong fields, heat essentially flows along the field lines, when $\boldsymbol{B}^{\text {tor }}$ is dominant $F_{\varphi}$ will also be much larger than $F_{\theta}$ and $F_{r}$ and produce a winding of the heat flow around the symmetry axis: $\boldsymbol{F}$ follows the shortest possible paths with the highest possible conductivity and this winding effectively acts as a heat blanket.

\section{Crustal temperature profiles}

In this section we will examine the temperature distributions resulting from a set of representative magnetic field configurations in which the three dipolar components $\boldsymbol{B}^{\text {crust }}, \boldsymbol{B}^{\text {core }}$, and $\boldsymbol{B}^{\text {tor }}$ have different relative stengths. We are faced with many parameters since beside the strengths of each component we have also the essentially unknown locations of the crustal currents sustaining the crustal fields, i.e., the two functions $s$ and $t$ of Eqs. (2)-(4), and. The cases of purely poloidal fields, i.e., $\boldsymbol{B}^{\text {tor }} \equiv 0$, with only $\boldsymbol{B}^{\text {crust }}$ or $\boldsymbol{B}^{\text {core }}$, but not both components superposed, were studied in Paper I and we will describe here a large set of models which, we hope, will allow us to get a good understanding of the joint effects of these, still simplified axisymmetric dipolar, multicomponent field configurations.

We consider first the case where the whole magnetic flux is produced in the crust and the field does not penetrate the core, i.e., $\boldsymbol{B}=\boldsymbol{B}^{\text {crust }}+\boldsymbol{B}^{\text {tor }}$ and $\boldsymbol{B}^{\text {core }} \equiv 0$. Figures 3 and 4 show the crustal temperature distributions, and the resulting surface temperatures, for two different locations of the toroidal components, with $x_{\max }=0.7$ and 0.2 , respectively, and in each case

3 This is a strong limitation of our simplifying assumption of axial symmetry and can only be removed by using a $3 \mathrm{D}$ code which, hopefully, will be studied in the near future. for three different strengths of $B_{0}^{\text {crust }}$. Panels a and b of Fig. 3 show that, given the high $T_{\text {core }}$, a $B_{0}^{\text {crust }}$ of $10^{12}$ or $10^{13} \mathrm{G}$ is not strong enough to affect the heat flow, in agreement with our previous results of Paper I: the inner part of the crust is essentially isothermal and it is the strong $\boldsymbol{B}^{\text {tor }}$ which act as a heat barrier, through the winding of the field lines in the $\varphi$ direction. With a very strong $\boldsymbol{B}^{\text {crust }}$ however, as in panel c of Fig. 3, the thermal insulation is then practically entirely produced by this component and $\boldsymbol{B}^{\text {tor }}$ is located too far out to play any noticable role. In Fig. 4 we illustrate the same cases of $\boldsymbol{B}^{\text {crust }}$ but with $\boldsymbol{B}^{\text {tor }}$ now located at higher densities: for $B_{0}^{\text {crust }}=10^{12}$ and $10^{13} \mathrm{G}$, panels a and $\mathrm{b}$, we see again that the thermal insulation is provided by the toroidal component but with the new effect that heat can flow "around" the toroidal field, from the magnetic axis toward the equator, in the outer part of the crust and result in much smaller meridional temperature gradients in this region. (This flow of heat "around" the toroidal field can also be seen in Fig. 3 but is much less efficient.) The case of a very strong $\boldsymbol{B}^{\text {crust }}$, panel c of Fig. 4, with this inner toroidal component is almost identical to the similar case of panel c of Fig. 3.

In the next two Figs. 5 and 6 we explore the effect of the distribution of the poloidal flux between the crust and the core, together with a toroidal component, i.e., we consider the three components $\boldsymbol{B}^{\text {crust }}, \boldsymbol{B}^{\text {core }}$, and $\boldsymbol{B}^{\text {tor }}$ together. We restrict ourselves to a $\boldsymbol{B}^{\text {tor }}$ whose maximum is located deep inside the crust, i.e., $\alpha=0.2$ or 0.5 in Eq. (12), which, as seen above, is not as effective as when located further out but is possibly more realistic since one expects currents to migrate toward the highest electrical conductivity layers (Page et al. 2000). In panels a and b of Fig. 5 the crust and the core contribute equally to the poloidal field and we see that when it is much weaker than the toroidal component, panel a, the latter acts as a heat barrier while in panel $\mathrm{b}$ the poloidal component is sufficiently strong to allow heat propagation in the radial direction, superposed of course to the winding flow in the $\varphi$ direction, along the field lines and only the region within about $30^{\circ}$ above and below the equator where the field lines are not connected to the core are slightly colder. In case the poloidal flux comes almost entirely 

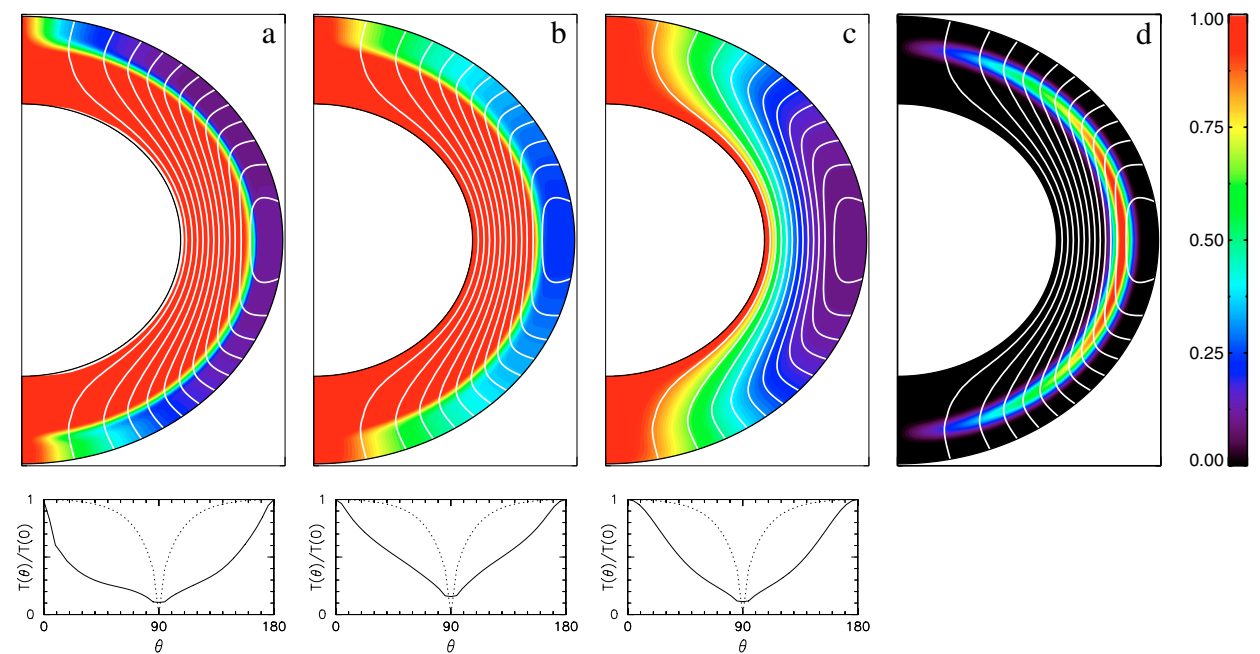

Fig. 3. Thermal structure of the crust of a neutron star. (The radial scale of the crust is stretched by a factor of 5 for clarity.) The magnetic field includes the $\boldsymbol{B}^{\text {crust }}$ and $\boldsymbol{B}^{\text {tor }}$ components and its structure is illustrated in panel d) (field lines of the poloidal component and color coded isolines of the toroidal component normalized on $B_{0}^{\text {tor }}$ ). The maximum of $B^{\text {tor }}$ is located at $x_{\max }=x_{\text {core }}+0.7 \Delta x_{\text {crust }}$ (see Eq. (12)). Panels a), b), and $\mathbf{c}$ ) show the temperature distribution for $B_{0}^{\text {tor }}=10^{15} \mathrm{G}$ and $B_{0}^{\text {crust }}=10^{12}, 10^{13}$, and $10^{14} \mathrm{G}$, respectively. The color coded temperatures are normalized on the core temperature, $T_{\text {core }}=6 \times 10^{7} \mathrm{~K}$. The lower panels show the resulting surface temperature profiles $T(\theta)$, once an extra insulating envelope has been glued to the core temperature distributions of the upper panels, and the dotted lines illustrate the same profile when an isothermal crust is assumed.
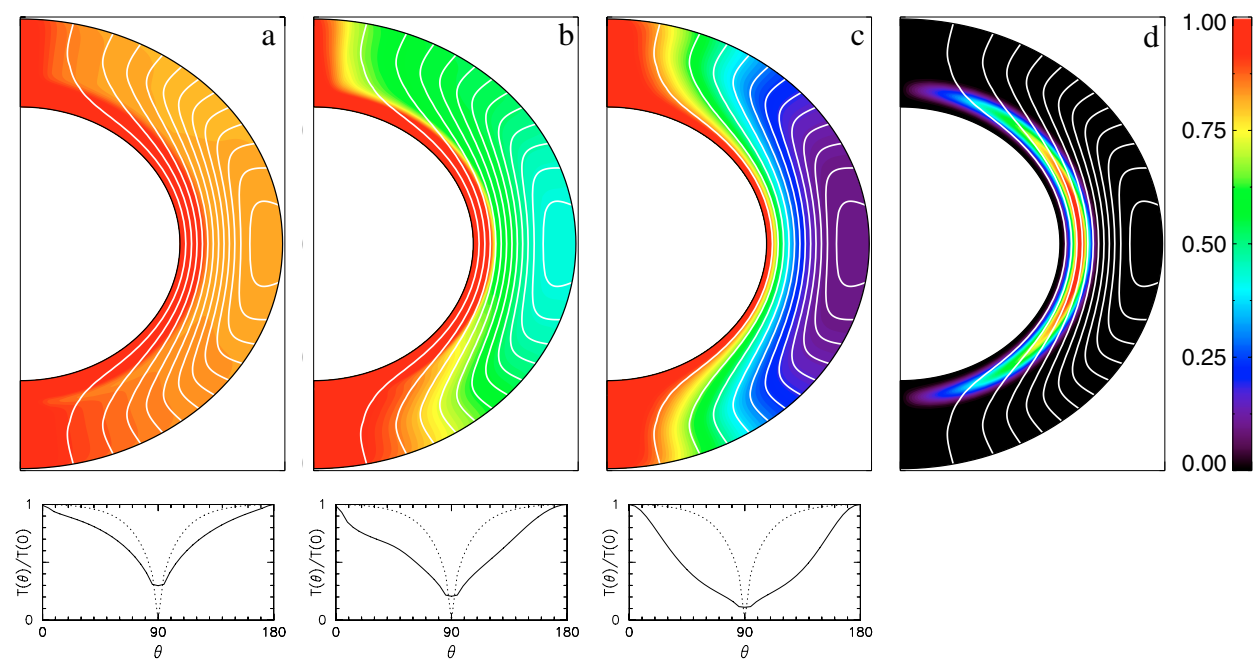

Fig. 4. The same as in Fig. 3 but assuming a location of the maximum of the toroidal component much closer to the crust-core interface: $x_{\max }=$ $x_{\text {core }}+0.2 \Delta x_{\text {crust }}$ (see Eq. (12)).

from the core, Fig. 5 panel d, the insulating effect of the toroidal field almost completely disappear. Assuming equipartition of the poloidal flux between the core and crust components, i.e. $\sim 10 \%$ coming from the crustal comonent, as in Fig. 5 panel c, the result is very similar to panel $\mathrm{c}$ despite of the fact that in this case the maximum of $\boldsymbol{B}^{\text {crust }}$ is about $1.3 \times 10^{13} \mathrm{G}$ (from its $\theta$ component, see Fig. 1), i.e., larger than $\boldsymbol{B}^{\text {core }}$.

We consider now an even stronger $\boldsymbol{B}^{\text {tor }}$ in Fig. 6 and see that its insulating effect is again very strong, due to the presence of the $\left(\omega_{B} \tau\right)^{2}$ in the denominators of Eq. (18) which is now increased by almost one order of magnitude. The results are perfectly in line with our description of the other previous cases: 1) the two models where the poloidal flux is crust dominated exhibit larger insulating effects than the corresponging models where the flux is core dominated, see panels a vs. $c$ and panels $b$ vs. d; and 2) models where the toroidal field is located deeper inside the crust are less insulating than when located further out, see panels b vs. a and panels d vs. c, since in the former cases heat can more easily flow arround the toroidal component back from the symmetry axis toward the equator.

A noticeable general feature of all our results is the asymmetry between the two magnetic hemispheres, resulting from the asymmetry of the total field $\boldsymbol{B}$, since $\boldsymbol{B}^{\text {crust }}$ and $\boldsymbol{B}^{\text {core }}$ are antisymmetric while $\boldsymbol{B}^{\text {tor }}$ is symmetric. In all cases where a nonisothermality is apparent the warm region close to the symmetry axis is broader in the lower hemisphere than in the upper one. In cases where the poloidal component is almost comparable to the toroidal one, as in panels c's of Figs. 3 and 4, the asymmetry is barely distinguishible but in all other cases it is clearly visible.

The lower panels of the four Figs. 3 to 6 show the resulting surface temperature profiles $T(\theta), \theta$ being the colatitude measured from the symmetry axis, scaled to the temperature at the magnetic pole $\theta=0$. For comparison, we also show, as dotted lines, the distribution resulting from an isothermal crust with magnetic field effect taken into account only in the envelope, according to PY01. These allow to easily produce the temperature 

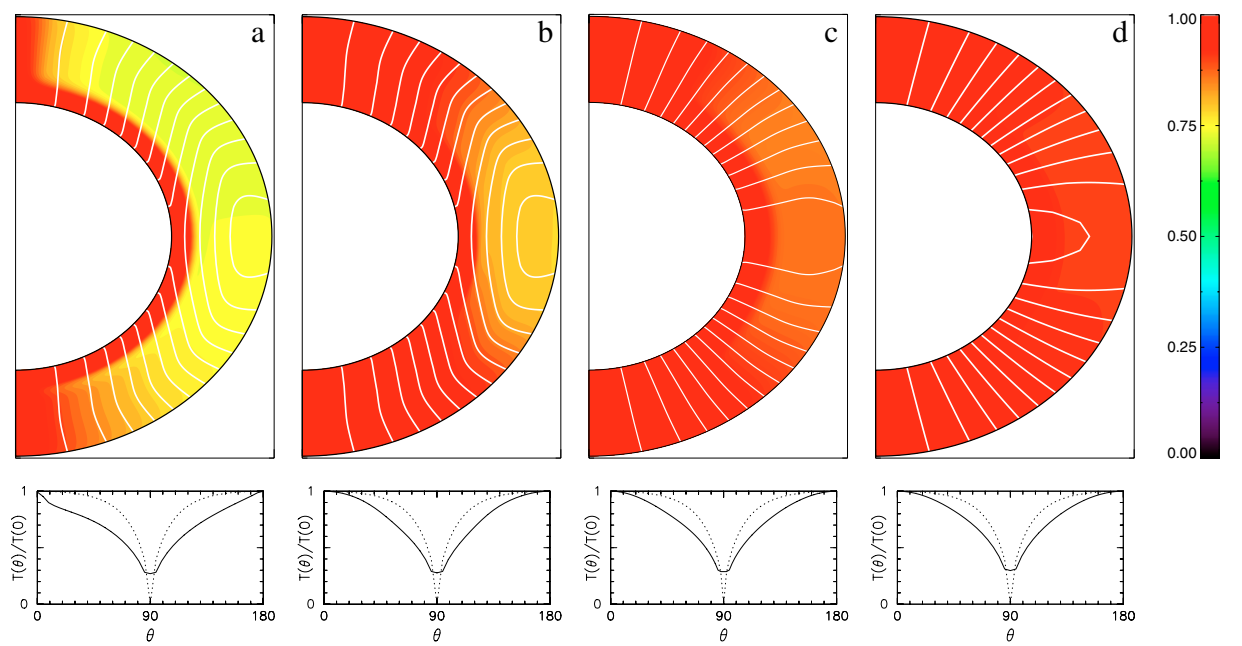

Fig. 5. The same as in Fig. 4 but now including the three components $\boldsymbol{B}^{\text {crust }}, \boldsymbol{B}^{\text {core }}$ and $\boldsymbol{B}^{\text {tor }}$. The magnetic field scales are in panel a) $B_{0}^{\text {crust }}=10^{12} \mathrm{G}$, $B_{0}^{\text {core }}=10^{12} \mathrm{G}$, panel b) $B_{0}^{\text {crust }}=10^{13} \mathrm{G}, B_{0}^{\text {core }}=10^{13} \mathrm{G}$, panel c) $B_{0}^{\text {crust }}=10^{12} \mathrm{G}, B_{0}^{\text {core }}=10^{13} \mathrm{G}$, and panel d) $B_{0}^{\text {crust }}=10^{11} \mathrm{G}, B_{0}^{\text {core }}=10^{13} \mathrm{G}$. In all four cases $B_{0}^{\text {tor }}=10^{15} \mathrm{G}$ and $x_{\max }=x_{\text {core }}+0.2 \Delta x_{\text {crust }}$ (see Eq. (12)).
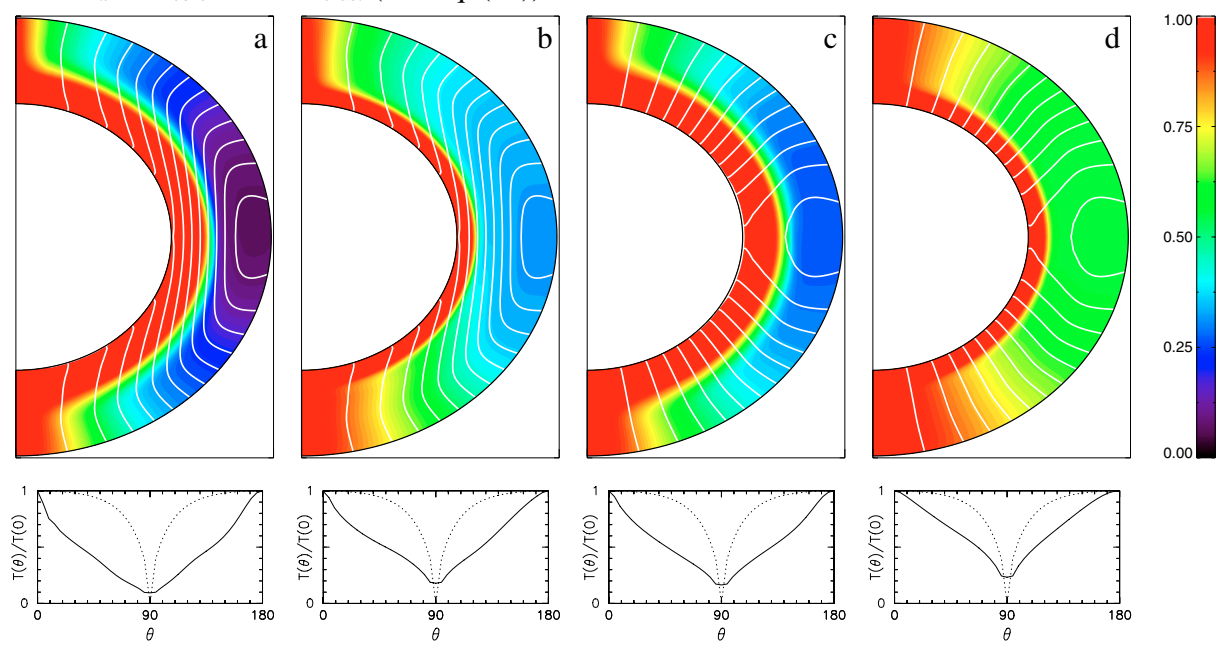

Fig. 6. The same as Fig. 5 but assuming a stronger toroidal field with $B_{0}^{\text {tor }}=3 \times 10^{15} \mathrm{G}$. In panels a and b the crustal poloidal field dominates the core field $\left(B_{0}^{\text {crust }}=7.5 \times 10^{12} \mathrm{G}, B_{0}^{\text {core }}=2.5 \times 10^{12} \mathrm{G}\right)$ while in panels c) and d) the core field is dominant $\left(B_{0}^{\text {crust }}=2.5 \times 10^{12} \mathrm{G}, B_{0}^{\text {core }}=7.5 \times 10^{12} \mathrm{G}\right)$. In panels a) and $\mathrm{c}$ the toroidal field has its maximum in the middle of the crust, $x_{\max }=x_{\text {core }}+0.5 \Delta x_{\text {crust }}$ (see Eq. (12)), while in panels b) and $\mathrm{d}$ it is located closer to the crust-core boundary $x_{\max }=x_{\text {core }}+0.2 \Delta x_{\text {crust }}$.

distribution over the whole NS surface and generate composite spectra and pulse profiles which we describe in the next section.

\section{Observational consequences for thermally emitting neutron stars}

The very distinct surface temperature distributions resulting from non-isothermal crusts as calculated in the previous section, and displayed in the lower panels of Figs. 3 to 6, have several immediate obervational consequences which we explore in this section. In all our simulations of spectra and pulse profiles we use simple isotropic blackbody emission at each point of the stellar surface, with the local temperature $T(\theta, \varphi)$, and include general relativistic light bending following the procedure described in Page (1995). Effects of strong magnetic fields on the emissivity and on the spectrum of the radiation are also significant and several models have already been studied (Pérez-Azorín et al. 2005; van Adelsberg et al. 2005; Ho \& Lai 2004). We do not take them into account in this work since it would result in a multiplication of the, already large, number of possible models and reserve their inclusion for a forthcoming paper.

In presence of a strong toroidal field in the crust, the channeling of heat toward the two polar regions results in the appearance of two hot spots of very reduced size. Figure 7 shows five examples of surface temperature distributions and the resulting observable pulse profiles. Naturally, models with the smallest hot spots result in the highest pulsed fractions, $P_{\mathrm{f}}$, with values above $30 \%$, in contradistinction to the case of an isothermal crust which results in $P_{\mathrm{f}} \sim 5 \%$.

The composite blackbody spectra resulting from the same five cases of Fig. 7 are shown in Fig. 8. Assumed distance to the stars have been adjusted to give the same maximum flux in the $\mathrm{X}$-ray band, and thus very similar X-ray spectra: given this adjustment the difference between the relative areas of the hot and cold regions in the various cases result in differences in the predicted optical fluxes. Comparison, by eye, of the surface temperature plots, left panels of Fig. 7, with the relative optical fluxes shows a direct correlation between the relative size of the cold region with the optical flux. This is confirmed by Fig. 9 in which the percentage of the total surface area whose temperature is 

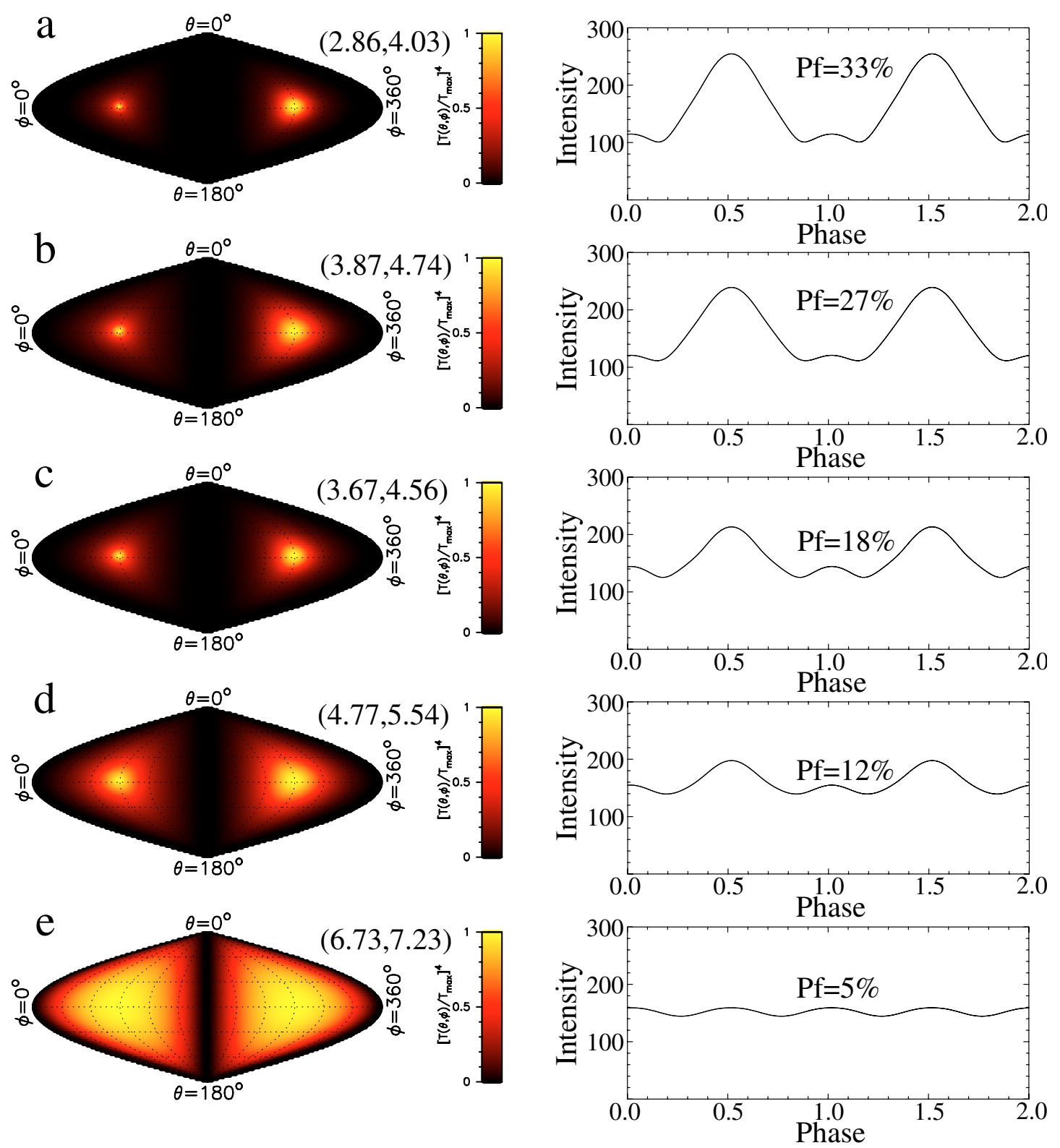

Fig. 7. Surface temperature distributions (left panels) in an area preserving representation with a color scale following the emited flux $\left(\propto T^{4}\right)$. Panels a to $d$ use the internal field structures of the corresponding panels in Fig. 6 while panel e assumes an isothermal crust. In all cases the dipolar symmetry axis is oriented in the rotational equatorial plane and the right panels show the resulting pulse profiles (in arbitrary units) which an observer, also located in the rotational equatorial plane, would detect. In all cases the core temperature is the same but the star's distance has been adjusted to give the same average flux (see Fig. 8). Number pairs within parentheses on the left panels give $\left(T_{\text {ave }}, T_{\text {eff }}\right.$ ), as defined in Eqs. (20) and (19), resp., in units of $10^{5} \mathrm{~K}$. All 5 models have almost the same maximum surface temperature $T_{\max } \simeq 8.45 \times 10^{5} \mathrm{~K}$ but different minimal temperatures $T_{\min }$.

below a certain value is shown and exhibit the same ordering as the optical fluxes of Fig. 8. This correlation can easily be made quantitative by noticing that most of the flux comes into X-ray and the latter is thus given by $F_{\mathrm{X}} \simeq a(R / D)^{2} \sigma_{\mathrm{SB}} T_{\text {eff }}^{4}, D$ being the star's distance and $a$ a correction factor for the interstellar absorption, where the effective temperature $T_{\text {eff }}$ is naturally defined by

$T_{\text {eff }} \equiv\left[\iint \frac{\mathrm{d} \Omega}{4 \pi} T_{\mathrm{s}}(\theta, \varphi)^{4}\right]^{1 / 4}$.

The optical flux, however, corresponding to the Rayleigh-Jeans tail of the spectrum is given by $F_{\mathrm{O}} \simeq(R / D)^{2} A T_{\text {ave }}, A$ being a constant dependent on the energy range included in the "optical", and the average temperature $T_{\text {ave }}$ being also naturally defined as

$T_{\text {ave }} \equiv \iint \frac{\mathrm{d} \Omega}{4 \pi} T_{\mathrm{s}}(\theta, \varphi)$

One hence has the simple relationship

$F_{\mathrm{O}} \simeq F_{\mathrm{X}} \frac{T_{\mathrm{ave}}}{T_{\mathrm{eff}}^{4}} \times \frac{A}{a \sigma_{\mathrm{SB}}}$.

The spectra plotted in Fig. 8 considered different distances $D$, adjusted to produce almost identical X-ray flux but with the same $N_{\mathrm{H}}$ and hence approximately the same $a$ 's, and Eq. (21) allows to correctly predict the relative $F_{\mathrm{O}}$ 's, within $10 \%$, using the values of $\left(T_{\text {ave }}, T_{\text {eff }}\right)$ given in Fig. 7. 


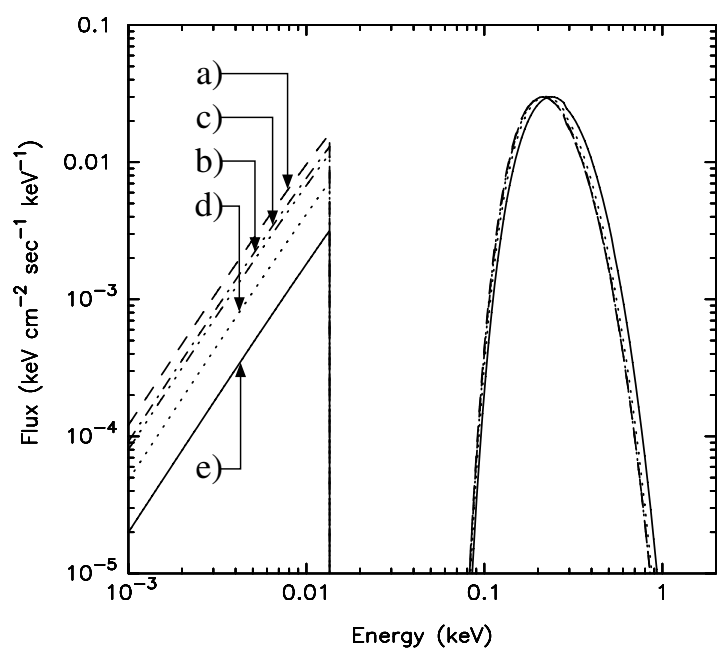

Fig. 8. Observable spectra for the five surface temperature distributions and observable pulse profiles, labelled as "a" to "e", shown in Fig. 7. The stars, with radii $R=11.4 \mathrm{~km}$ and $R_{\infty}=14.28 \mathrm{~km}$ for a $1.4 M_{\odot}$, are assumed to be at distances of 100,142, 131, 202, and 220 pc, resp., to produce almost identical observable spectra in the X-ray band $\left(N_{\mathrm{H}}=\right.$ $1 \times 10^{20} \mathrm{~cm}^{-2}$ for interstellar absorption) but resulting in significantly different fluxes in the optical range.

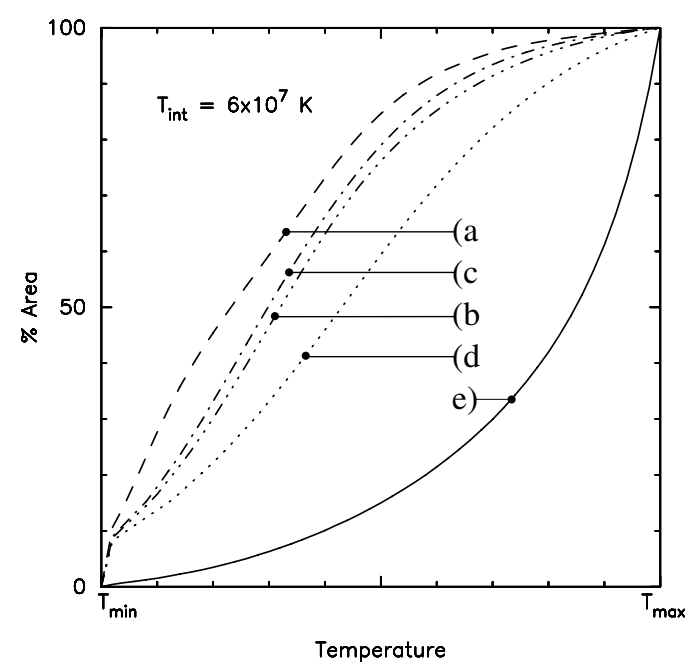

Fig. 9. Distribution of the surface temperature in terms of the percentage of the superficial area whose temperature is lower than $T$, for the five models of Figs. 7 and 8.

As a concrete example, we show in Fig. 10 an attempt to model the spectrum of the isolated NS RX J1856.5-3754. Its X-ray spectrum is well fitted by a hot blackbody, with $T^{\infty} \sim$ $6-7 \times 10^{5} \mathrm{~K}$ and an effective radius $R_{\mathrm{H}}^{\infty} \sim 4.5 \mathrm{~km}$, while the optical data can be interpreted as the Rayleigh-Jeans tail of a cold blackbody component with $T^{\infty} \leq 3.5 \times 10^{5} \mathrm{~K}$ and an effective radius $R_{\mathrm{C}}^{\infty} \geq 17 \mathrm{~km}$, assuming a distance $D=117 \mathrm{pc}$ (Pons et al. 2002; Trümper et al. 2004) which are plotted in this figures as dashed lines. Using the field structure of panel $\mathrm{c}$ of Fig. 6 and the resulting surface temperature profile we obtain a reasonable fit, shown as a continuous line, to this two blackbodies description. In contradistiction to the models presented in Fig. 7 where both the magnetic field axis and the observer's line of sight were chosen to be perpendicular to the rotational axis in order to obtain the largest possible pulsed fraction, in the case of RX J1856.5-3754 the upper limit on the pulsed fraction of $1.3 \%$ (Burwitz et al. 2003) requires some alignement of either

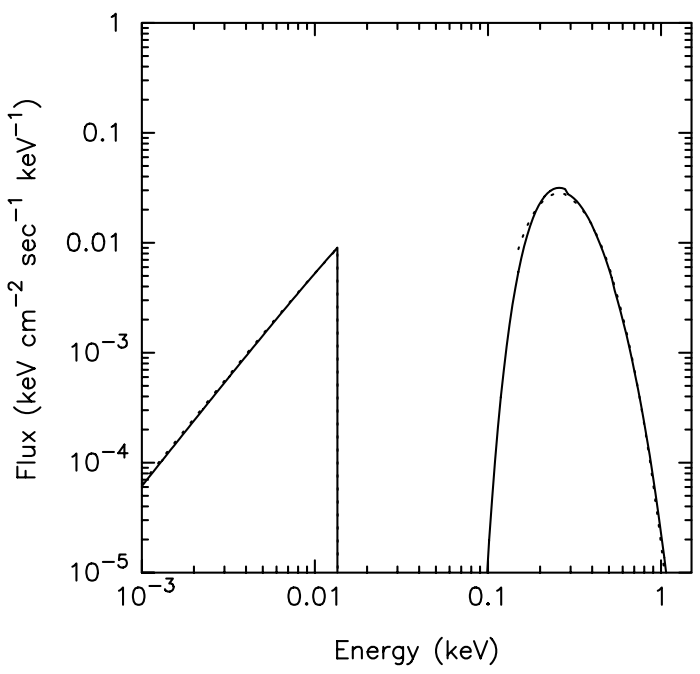

Fig. 10. Fit of the spectrum of RX J1856.5-3754. Dotted lines show the two blackbodies fit to the data from Trümper et al. (2004). The continuous line show our results: the star has a radius $R=14.4 \mathrm{~km}$ and $R_{\infty}=17.06 \mathrm{~km}$ for a $1.4 M_{\odot}$, at a distance of $122 \mathrm{pc}\left(N_{\mathrm{H}}=1.6 \times\right.$ $10^{20} \mathrm{~cm}^{-2}$ for interstellar absorption) and the observer is assumed to be aligned with the rotation axis. The magnetic field structure corresponds to model c of Fig. 6 adjusted to the $14.4 \mathrm{~km}$ radius with $T_{\mathrm{b}}=6.8 \times 10^{7} \mathrm{~K}$, resulting in $T_{\text {eff }}^{\infty}=4.62 \times 10^{5} \mathrm{~K}$ and $T_{\max }^{\infty}=8.54 \times 10^{5} \mathrm{~K}$.

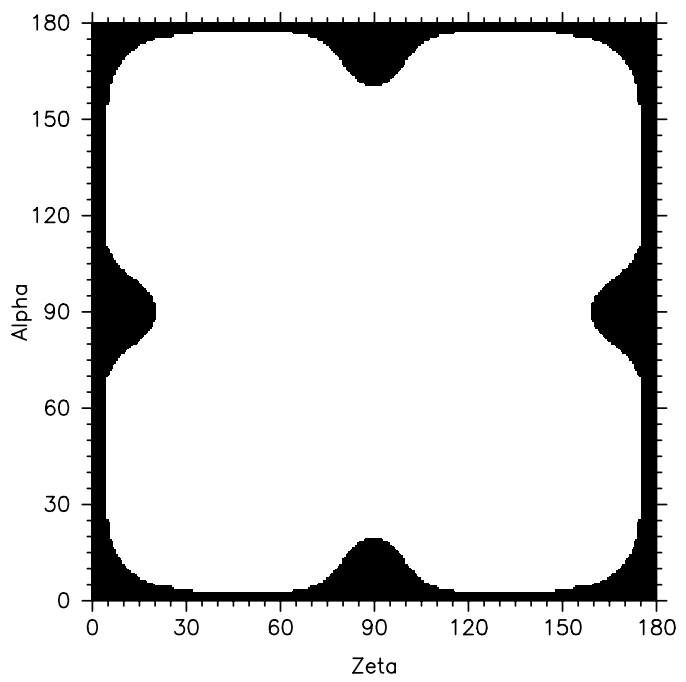

Fig. 11. Representation of the allowed region in the $\alpha-\zeta$ plane, $\alpha$ and $\zeta$ being the angle between the observer's direction and the magnetic axis, respectively, with the rotational axis for which the surface temperature model of Fig. 10 results in observable pulsed fraction lower than $1.3 \%$. For each pair of $\alpha$ and $\zeta$ pulse profiles were calculated as described in Page (1995), including GR effects of light bending for a $R=14.4 \mathrm{~km}$, $1.4 M_{\odot}$, star, and assuming, from each point of the surface, isotropic blackbody emission at the corresponding temperature. Pairs resulting in a $P_{\mathrm{f}}$ smaller than $1.3 \%$ are marked in black and their joint probability is about $10 \%$.

the observer or the magnetic field axis with the rotational axis. We quantify the allowed range of these two angles in Fig. 11 from which we deduce a probablilty of about $10 \%$ for such a geometry to be realized.

\section{Discussion and conclusions}

We have studied the impact of different crustal magnetic field configurations on the transport of heat within the crust of a NS 
and the resulting surface temperature distributions with their observable consequences. In a previous paper (Paper I) we had first considered the simplest cases of a poloidal dipolar field either entirely confined to the crust ("crustal field") or exclusively produced by currents in the core and hence having in the crust the same structure as a vacuum dipole ("core field"). In the present work we have considered the effect of an additional (dipolar) toroidal component, located within the crust, with various combinations of core and crustal fields, and all three components having the same symmetry axis.

Since we consider here only poloidal and toroidal dipolar components, due to the axial symmetry the toroidal field component is confined to the interior of the NS and the external field geometry is always a purely poloidal dipolar one uniquely characterized by its strength at the magnetic pole, $B_{0}$. Therefore, the effects of various magnetic field structures with the same $B_{0}$ in the magnetosphere, as e.g. pulsar properties and rotational evolution of the NS, are indistinguishable. However, these different inner geometries of the magnetic field result in very different surface temperature distributions which are potentially distinguishable through observations of thermal emission from isolated cooling NSs.

We had shown in Paper I that a strong magnetic field practically channels the heat flow along its field lines. We had found that a core field, with field lines essentially radial in most of the crust, results in an almost isothermal crust and the surface temperature is hence only controlled by the anisotropic transport in the uppermost layers of the envelope (Greenstein \& Hartke 1983; Page 1995) exhibiting two symmetric extended warm regions, one in each hemisphere, and a cold belt along the magnetic equator. In contradistinction, a crustal field, having field lines with large meridional components, can produce strongly non-isothermal crusts and the heat flow is much more concentrated around the magnetic poles, resulting in a much wider cold equatorial belt. Our main result here is that the addition of a toroidal component, inside the crust, is able to add a very efficient heat blanket which may force the heat to flow within a narrow region along the polar axis and result in a cold region covering most of the stellar surface with two warm spots arround the two magnetic poles. However, we find that for this effect to occur it is essential to have a significant part of the poloidal field produced in the crust: the toroidal field in itself is not sufficient in case the field lines of the poloidal component are almost radial.

The presence of two small warm regions separated by an extended cold belt has two inmediate observational consequences. The first is that the observable pulsed fraction in the X-ray band can be very large, above $30 \%$ assuming isotropic blackbody emission, and, second, the cold region's emission contributes little to the X-ray flux but dominates the detectable flux in the optical range, appearing as an "optical excess". Notice, however, that addition to the dipolar poloidal field of a quadrupolar component also allows, potentially, to reach high pulsed fractions (Page \& Sarmiento 1996) within the same isotropic blackbody emission scheme and without any todoidal component. In this latter case, high $P_{\mathrm{f}}$ 's are reached when the quadrupole sufficiently deforms the field to push the two magnetic poles close to each other but without significantly altering the star's effective and average temperature (see, e.g., Fig. 1 of Page \& Sarmiento 1996), and hence producing no significant "optical excess". The "Magnificent Seven" briefly presented in the Introduction seem to be very good candidate NSs for which our results may be relevent. Their estimated dipolar surface field strengths $B_{0}$ are above $10^{13} \mathrm{G}$, opening the possibility of strong anisotropic heat transport in their crust, and the "optical excess" observed in
5 cases is then a natural consequence of the large $B_{0}$ 's in case their crusts harbor very strong poloidal fields. As we has seen, channeling of the heat flux toward two small polar regions requieres moreover that part of the poloidal flux be confined to the crust: this can be achieved either by the action of some dynamo process which produced such a poloidal field component or/and by the expulsion of flux from the core through the action of the outward migration of the neutron superfluid fluxoid resulting from the NS spin-down and the consecutive expulsion of the magnetic fluxoid resulting from the core proton superconductor. This latter process is likely to have occured in the "Magnificent Seven" given their unusually long period, for their relatively young ages, which are very likely the results of fast spin-down due to their strong dipolar poloidal fields. However, our axisymmetric field configurations produce symmetric, but not sinusoidal, light-curves and the superposition of a quadrupolar component may be necessary to produce precise fits of the observed pulse profiles of the seven XDINS (Zane \& Turolla 2005)

Results very similar to the ones presented here have been recently obtained by Pérez-Azorín et al. (2006) who also conclude that a toroidal component localized within the crust lead to surface temperature distributions with very localized warm regions and an extended cold equatorial belt. These authors also took into account the transport of heat by phonons, which is unaffected by the magnetic field and thus reduces the anisotropicity, and the small quantum corrections present at high densities for very strong fields, two effects we did not consider. Moreover, they performed their heat transport calculations to lower densities than we have considered here and assumed that the upper layer is cut at a finite density due to solidification of the material while we simply assumed that the envelope extends into an atmosphere and "glued" our models with pre-existent envelope models. Nevertheless, despite of these differences, the similarity of the results prove that the effect of a toroidal field is independent of the details and make our common results very robusts.

Claiming that the observed optical-X-ray properties of the "Magnificent Seven" prove the existence of strong toroidal fields in their crust may still be premature. Given our poor understanding of the emissive properties of the NS surface, being it either an atmosphere, a liquid or a solid depending on its chemical composition, temperature and magnetic field strength, one cannot exclude that the "optical excess" is achieved locally, i.e., is an intrinsic feature of the spectrum produced at each point of the surface, instead of being a global effect due to strong surface temperature differences as we have obtained. In contradistinction, three very active pulsars exhibit very different properties (Vela: Romani et al. 2005, 2005; PSR B0656+14: Shibanov et al. 2005; and Geminga: Kargaltsev et al. 2005): a thermal spectrum is clearly seen in the X-ray but near \& far UV observations give upper limits on its Rayleigh-Jeans tail which are in agreement or even below the extrapolations of the X-ray spectrum. The reasons for this different behavior is presently unknown and may be due to their weaker magnetic field, different surface chemical composition, and/or reprocessing of the thermal photons in the magnetosphere.

The "Magnificent Seven" have temperatures and/or age estimates which definitely place them at the photon cooling stage (see, e.g., Page et al. 2006), i.e. their evolution is driven by energy loss from the surface emission. The large reduction in the surface photon luminosity resulting from our crustal field configurations, which is clearly seen from Fig. 7, will have the immediate consequence that the cooling will proceed much more 
slowly. A detailled study of this effect is under study and will presented in a forthcoming paper.

Finally, an issue raised by our models is the time evolution and stability of the field configurations we have considered. We intend to perform coupled time-dependent cooling and field evolution calculations to try to eliminate at least the most unlikely configurations, i.e., either highly unstable configurations and/or configurations based on current distribution which will evolve fast and reajust or decay. Nevertheless we can speculate that a very strong crustal toroidal field could produce such strong tension that the crust should readjust itself, as is seen in magnetars, and this may be the cause of the observed change in the spectrum and pulse profile of RX J0720.4-3125 (de Vries et al. 2004).

Acknowledgements. Part of this work is supported by a binational grant from DGF-Conacyt \#444MEX113/4/0-2. D.P.'s work is partially supported by grants from UNAM-DGAPA (\#IN112502) and Conacyt (\#36632-E). Discussions with F. Haberl, J. Pons and J. Trümper are gratefully acknowledged.

\section{References}

Akiyama, S., Wheeler, J. C., Meier, D. L., \& Lichtenstard, I. 2003, ApJ, 584, 954

Akmal, A., Pandharipande, V. R., \& Ravenhall, D. G. 1998, Phys. Rev., C, 58, 1804

Balbus, S. A., \& Hawley, J. F. 1991, ApJ, 376, 214

Balbus, S. A., \& Hawley, J. F. 1998, Rev. Mod. Phys., 70, 1

Braithwaite, J., \& Spruit, H. 2004 Nature, 431, 819

Brinkmann, W. 1980, A\&A, 82, 352

Buckley, K. B. W., Metlitski, M. A., \& Zhitnitsky, A. R. 2004, Phys. Rev. Lett., 92, 151102

Burwitz, V., Haberl, F., Neuhauser, R., et al. 2003, A\&A, 399, 1109

Chandrasekhar, S. 1961, Hydrodynamic and Hydromagnetic Stability (Oxford University Press)

de Vries, C. P., Vink, J., Méndez, M., \& Verbunt, F. 2004, A\&A, 415, L31

Geppert, U., \& Urpin, V. 1994, MNRAS, 271, 490

Geppert, U., \& Wiebicke, H.-J. 1986, Ap\&SS, 128, 281

Geppert, U., Küker, M., \& Page, D. 2004, A\&A, 426, 267

Gil, J., Melikidze, G., \& Geppert, U. 2003, A\&A, 407, 315
Greenstein, G., \& Hartke, G. J. 1983, ApJ, 271, 283

Haberl, F. 2004a, AdSpR, 33, 638

Haberl, F. 2004b, Mem. Soc. Astron. It., 75, 454 [arXiv: astro-ph/0401075]

Haberl, F. 2005 [arXiv:astro-ph/0510480]

Heyl, J., \& Hernquist, L. 1998, MNRAS, 300, 599

Ho, W., \& Lai, G. 2004, ApJ, 607, 420

Kargaltsev, O., Pavlov, G. G., Zavlin, V. E., \& Romani, R. W. 2005, ApJ, 625, 307

Kluźniak, W., \& Ruderman, M. 1998, ApJ, 505, L113

Lai, D. 2001, Rev. Mod. Phys., 73, 629

Link, B. 2003, Phys. Rev. Lett., 91, 101101

Markey, P., \& Tayler, R. J. 1973, MNRAS, 163, 77

Motch, C., Zavlin, V., \& Haberl, F. 2003 A\&A, 408, 323

Page, D. 1995, ApJ, 442, 273

Page, D., \& Sarmiento, A. 1996, ApJ, 473, 1067

Page, D., Geppert, U., \& Zannias, T. 2000, A\&A, 360, 1052

Page, D., Geppert, U., \& Weber, F. 2006, Nucl. Phys. A, in press [arXiv:astro-ph/0508056]

Pérez-Azorín, J. F., Miralles, J. A., \& Pons, J. A. 2005, A\&A, 433, 275

Pérez-Azorín, J. F., Miralles, J. A., \& Pons, J. A. 2006, A\&A, 451, 1009

Pons, J. A., Walter, F. M., Lattimer, J. M., et al. 2002, ApJ, 564, 981

Potekhin, A. Y., \& Yakovlev, D. G. 2001, A\&A, 374, 213 (PY01)

Potekhin, A. Y., Yakovlev, D. G., Charbier, G., \& Gnedin, O. Y. 2003, ApJ, 594, 404

Rädler, K.-H. 2000, in From the Sun to the Great Attractor, Lect. Notes in Phys. 556, ed. D. Page, \& J. G. Hirsch (Springer), 101

Rädler, K.-H., \& Geppert, U. 1999, Workshop on Stellar Dynamos, ed. M. Nunez, \& A. Ferriz-Mas, ASP Conf. Ser., 178

Romani, R. W., Kargaltsev, O., \& Pavlov, G. G. 2005, ApJ, 627, 383

Schwope, A. D., Hambaryan, V., Haberl, F., \& Motch, C. 2005, A\&A, 441, 597

Shibanov, Yu. A., Sollerman, J., Lundqvist, P., Gull, T., \& Lindler, D. 2005, A\&A, 440, 693

Thompson, C., \& Duncan, R. C. 1993, ApJ, 408, 194

Trümper, J., Burwitz, V., Haberl, F., \& Zavlin, S. 2004, NuPhS, 132, 560

Turolla, R., Zane, S., \& Drake, J. R. 2004, ApJ, 603, 265

van Adelsberg, M., Lai, D., Potekhin, A., \& Arras, P. 2005, ApJ, 628, 902

Ventura, J., \& Potekhin, A. 2001, in The Neutron Star - Black Hole Connection, ed. C. Kouveliotou, J. Ventura, \& E. Van den Heuvel (Kluwer Academic Publishers), 393 [arXiv: astro-ph/0104003]

Wheeler, J. C., Meier, D. L., \& Wilson, J. R. 2002, ApJ, 568, 807

Yakovlev, D., \& Kaminker, A. 1994, in The Equation of State in Astrophysics, ed. G. Chabrier, \& E. Schatzman (Cambridge University Press), 214

Zane, S., \& Turolla, R. 2005, MNRAS, 366, 727 